Vol. $7, n^{\circ} 1 \mid 2003$

Varia

\title{
Idées, institutions, usages, savoirs : l'esprit de la justice, la sagacité de l'expert, la puissance du droit de punir
}

Michel Porret

\section{(2) OpenEdition \\ Journals}

Édition électronique

URL : https://journals.openedition.org/chs/617

DOI : $10.4000 /$ chs. 617

ISSN : 1663-4837

Éditeur

Librairie Droz

\section{Édition imprimée}

Date de publication : 1 juillet 2003

Pagination : 129-138

ISBN : 2-600-00865-9

ISSN : 1422-0857

\section{Référence électronique}

Michel Porret, « Idées, institutions, usages, savoirs : l'esprit de la justice, la sagacité de l'expert, la puissance du droit de punir », Crime, Histoire \& Sociétés / Crime, History \& Societies [En ligne], Vol. $7, n^{\circ} 1$ 2003, mis en ligne le 24 février 2009, consulté le 23 mars 2022. URL : http://journals.openedition.org/ chs/617 ; DOl : https://doi.org/10.4000/chs.617

Ce document a été généré automatiquement le 23 mars 2022.

(C) Droz 


\title{
Idées, institutions, usages, savoirs : l'esprit de la justice, la sagacité de l'expert, la puissance du droit de punir
}

\author{
Michel Porret
}

1 Revues spécialisées, congrès internationaux, journées d'études à l'Université, « Rendezvous de l'Histoire" ouverts au grand public, ouvrages de synthèses, monographies érudites, thèses, livres collectifs : l'histoire culturelle, institutionnelle et sociale de la justice et $\mathrm{du}$ crime est aujourd'hui un champ autonome et prolixe dans l'historiographie contemporaine. À sa manière, Crime, Histoire et Sociétés illustre cette vitalité historiographique par les articles inédits que la revue publie et par les nombreux ouvrages scientifiques qu'elle reçoit des quatre coins de la planète. Ils constituent une bibliothèque vaste et passionnante, autant pour l'Ancien Régime que pour l'époque contemporaine.

2 Les rendez-vous de l'histoire, Le Crime et le pouvoir, Blois 1998 (Éditions Pleins feux, Nantes, 1999, 126 pages), avant-propos de Francis Chevrier, préface de Maurice Sartre. De manière parfois dissonante, les historiens contemporanéistes, Jean Lacouture, Dominique Kalifa, Marc Ferro, Jean-Luc Domenbach et René Rémond, affrontent la vaste problématique du crime et du pouvoir politique dans des objets aussi éclatés que la figure de Montaigne confronté au mal, le crime et les polices privées ou d'État à la «Belle Époque ", le crime et le pouvoir en Chine au XX siècle, les crimes d'État dans l'Allemagne nazie et l'URSS, les liens entre l'Église catholique et le nazisme. Depuis le 11 septembre 2001, la réponse que l'État de droit apporte au crime politique qu'est l'hyper-terrorisme actualise cette réflexion commune bien remise en perspective par René Rémond. Dans un texte conclusif, "Le Crime face à l'histoire ", il souligne justement que l'histoire du crime politique reste théoriquement un objet sans avenir dans un État de droit qui remplace l'action directe par la loi, la violence par le débat parlementaire, la vengeance privée par la justice. Ce petit volume ouvre maintes pistes 
de recherches sur l'histoire de l'État moderne et contemporain dans la constitution de sa légitimité. Montrant que la notion de crime politique est souvent tolérée et évolutive (tyrannicide, crime de lèse-majesté, régicide, terrorisme, etc.), il invite à une réflexion comparative plus soutenue sur l'histoire de la culture du crime politique. Motivés dès l'Antiquité par la raison d'État, les crimes politiques sont à l'ordre du jour durant les grandes révolutions (française, soviétique, maoïste, etc.). Les régimes totalitaires s'appuient sur le crime politique pour détruire les opposants (assassinat de Léon Trotsky au Mexique en 1940). Contre les populations civiles notamment, la guerre l'utilise pour arriver à ses fins coloniales ou hégémoniques. Crime politique, crime de guerres, crime contre l'humanité : est-ce là la même histoire du mal absolu, culminant au $\mathrm{XX}^{\mathrm{e}}$ siècle, qu'évoque ce petit ouvrage? Un mal condamné par des cours pénales internationales aussi différentes que le tribunal de Nuremberg ou celui de la Haye. Si le pouvoir génère souvent le crime politique, l'État détenteur du droit ne peut que le combattre s'il veut survivre. Vaste champ pour les historiens du droit de punir.

3 Le Temps de l'histoire, sous la direction de Frédéric Chauvaud (Université de Poitiers), Histoire et justice, panorama de la recherche, novembre 2001, 266 pages $^{1}$. Ouverte par un bilan de l'historiographie française, esquissé par Jean-Claude Farcy (de l'« école des Annales » à la micro-histoire des années 1990), qui favoriserait aujourd'hui l'étude sociale du crime et de la prison plutôt que celle des institutions judiciaires, une première section de cette livraison, fruit d'une table ronde d'archivistes, de chercheurs et d'historiens tenue à l'Université de Poitiers en mars 2000, illustre l'« histoire de la justice en construction " pratiquée dans plusieurs centres de recherches (Mission de recherche droit et justice; Association française pour l'histoire de la Justice; CNFE -PJJ; CESDIP; Universités : Angers, Dijon, Genève, Grenoble, Limoges, Poitiers). La seconde partie du dossier publie douze études inédites sur l'histoire culturelle et sociale de la justice, de l'Antiquité à l'époque contemporaine : complaintes judiciaires sous l'Ancien régime à Paris; plaidoyers des avocats sophistes à Rome au IV ${ }^{\mathrm{e}}$ siècle; débat de 1908 sur la peine de mort; figure du juge philanthrope à l'aube de 1900; qualification du parricide dans la pratique pénale française du XIXe siècle; justice en caricature dans le Père Peinard (1889-1902); justice royale au XV ${ }^{\mathrm{e}}$ siècle; travail des juges sous Louis XIII; sociabilité dans le ressort du présidial de Reims au XVIII ${ }^{\text {e }}$ siècle; notariat et infrajudiciaire à Limoges (1735-1740); usage des monitoires dans le diocèse de Poitiers; vol par «nécessité » au XIXe siècle. Multicolore, cette palette de monographies pointues illustre les "sensibilités et les approches différentes" qui donnent un sens contemporain à l'histoire vivante de la justice et du crime et ouvrent de nouvelles pistes de recherches.

4 Travaillant dans le vaste champ la "médecine du crime $»^{2}$, Philippe Artières (CNRS) dessine des objets particulièrement originaux dans l'histoire des savoirs positifs liés à la justice dès l'aube du XIX ${ }^{e}$ siècle. Avant son récent (2002), Artières (1998) consacrait sa thèse à la problématique de la constitution médicale de l'écriture des déviants en objet de vérité : 270 pages, notes in fine, hélas dépourvue de tout index onomastique et de toute bibliographie finale. Ouvert par une "préface» anonyme et un pertinent plaidoyer («Introduction») pour l'histoire culturelle de l'expertise psychiatrique et médico-légale, l'ouvrage est divisé en neuf chapitres bouclés par des "Conclusions » évoquant la dimension créatrice de l'écriture (Antonin Artaud, Raymond Roussel). Finement écrit au plus près des sources médico-légales qu'Artières domine, ce livre donne à penser un nouvel objet de l'histoire des savoirs et des usages de la criminologie : la « science de l'écriture » qui se développe en France entre 1870 et 1914 
avec l'émergence des experts graphologues. Dès la Renaissance, induite par la procédure inquisitoire (écrite, secrète, système de preuves basé sur l'aveu et la matérialité des preuves concrètes), l'expertise judiciaire des corps, des objets et des écritures seconde les juges du siège. Elle culmine au XIX ${ }^{e}$ siècle, sous le double effet social et institutionnel de la légalité pénale et du positivisme scientifique. Son histoire dessine un vaste champ d'investigation historique. Médecine légale, criminologie, anthropologie et psychiatrie criminelles : ces savoirs normatifs placent le judiciaire et le droit de punir dans l'orbite des sciences naturelles et expérimentales telles qu'elles ont été mises en place par les Lumières. Cesare Lombroso (1835-1909), Alexandre Lacassagne (1843-1924), Ambroise-Auguste Tardieu (1838-1879) ou encore Rodolphe A. Reiss (cf.infra) : après 1850, l'internationale criminologique domine le champ pénal dans toute l'Europe. Les « experts du crime » veulent quantifier le passage à l'acte criminel pour prévenir la récidive. Ils modélisent les invariants de la personnalité délinquante. Ils classent les "circonstances" morales et matérielles du crime pour objectiver le mode opératoire. Dans cette finalité expertale, l'« écriture des déviants » est un enjeu primordial pour l'étiologie criminelle positiviste. Comme le langage du «corps déviant» qu'est le tatouage de l'homo criminalis (Lacassagne à Lyon), l'«écriture ordinaire " révèle la pathologie humaine qui occupe le champ normatif du médicolégal. Privilégiant la rupture entre les pratiques de l'Ancien régime et celles «modernes» du XIX ${ }^{e}$ siècle (les archives judiciaires de l'Ancien régime regorgent d'expertises en écritures qui fondent une "graphologie" morale et empirique), Artières étudie la constitution et l'usage de ce «regard spécifique sur l'écriture » du criminel, de l'aliéné, de l'« anormal». Si au fil du XIXe siècle une mutation culturelle des techniques d'écriture atténue l'individualité (sténographie, machine à écrire), la "médicalisation de l'écriture» recoupe trois enjeux épistémologiques: triple dimension de l'écriture (écriture échappant à la grille de lecture classique, signes graphiques témoignant du caractère anormal du scripteur, gestes graphiques révélant des pathologies inconnues); formation d'un savoir autonome sur l'écriture; prolongement socio-professionnel de la graphologie dans le champ psychiatrique et judiciaire. De même que les érudits du XVII ${ }^{e}$ siècle distinguaient les bonnes et les mauvaise chartes, la graphologie des modernes doit discerner le vrai du faux. Autour du discours de l'«absent» (aliéné, criminel, suicidé), utilisant la phrénologie ou la psychiatrie morale, la clinique de l'écriture sonde les billets des suicidés, les écrits des aliénés, les palimpsestes des prisons. Les cliniciens de la graphie suscitent des autobiographies d'incarcérés : prisonniers de droit communs, aliénés. L'écriture est donc captive d'un espace carcéral ou asilaire et du protocole scientifique de sa production. Exigée pour l'enquête psychiatrique ou judiciaire, l'écriture de l'aliéné ou $\mathrm{du}$ prisonnier est source de souffrance disciplinaire. Prenant la graphie comme symptôme, la volonté de savoir des experts érige l'écriture comme le signe de la déviance, comme le miroir déformant de l'anormalité. Avec notamment AlexandreJacques-François Brierre de Boismont (1798-1881), les aliénistes éditent les écrits de leurs patients pour "lire dans la pensée des fous", puis modéliser des types pathologiques. L'écriture devient l'étalon idéal des normes et des déviances. Les pédagogues diagnostiquent la " bonne » ou " mauvaise » discipline des élèves dans leur écriture lâche ou dominée. Les criminologues examinent l'« écriture des canailles » pour penser le crime et son milieu³. En 1897, pour Gabriel Tarde, l'écriture est la clef d'analyse des rapports sociaux. Les médecins légistes l'utilisent pour résoudre le problème des testaments contestés. Elle devient un enjeu technique: les chimistes 
rêvent de revivifier des encres anciennes (écritures effacées ou ajoutées). Le sexe, l'âge, l'intelligence, le sens moral: tels sont les éléments que les psychologues recherchent dans l'« 'écriture ordinaire ». Dès 1870 , mise dans un processus d'objectivation de la vérité, l'écriture occupe ainsi des champs de savoirs entremêlés: médecine légale, criminologie, chimie, neurologie, psychiatrie, hygiène sociale. Or, l'expertise graphologique reste incertaine. Elle contient en outre un enjeu politique illustré par la querelle des experts durant l'affaire Dreyfus (1894-1906). Les déchiffrements d'un bordereau manuscrit d'abord attribué fautivement à Dreyfus (notamment par Alphonse Bertillon, «inventeur» de l'anthropométrie criminelle) l'accusent. De nouvelles expertises graphologiques innocentent le capitaine déporté et ouvrent la voie à sa lente réhabilitation. Ancrée ainsi dans le juridique, la clinique de l'écriture dessine le champ du normatif: en outre, elle offre aux sujets humains de l'expertise la capacité de se raconter («Les Aliénés peints par eux-mêmes » dans L'Encéphale, 1886, du docteur Emmanuel Régis, spécialiste de la psychiatrie du régicide et introducteur de Freud en France dès 1913). En constituant ainsi des "cas médicaux" pour objectiver les pathologies et considérer la subjectivité des aliénés et des criminels, la clinique de l'écriture place les individus, les mots et les choses dans les catégories du vrai ou du faux, du mal ou du bien, du normal ou du pathologique. Dès 1900, les aliénistes et les légistes qui valorisent ainsi l'écriture de leurs patients (leurs dessins aussi) constituent l'« archive mineure » d'une nouvelle esthétique littéraire. Celle du délire ou celle de l'autobiographie criminelle soumises à la "frénésie graphologique » qui occupe alors les esprits. En inventant ainsi l'« art pathologique » menant à l'« art brut », la clinique de l'écriture apporte une touche culturelle considérable à la modernité sociale du $\mathrm{XX}^{\mathrm{e}}$ siècle en insistant sur l'individualité révélée à charge par l'écriture des déviants.

Pas de considérations esthétisantes sur le crime chez le «pionnier de la police scientifique » Rodolphe Archibald Reiss né à Hechtsberg (Allemagne) en 1875, citoyen suisse dès 1901, mort à Belgrade en 1929 après avoir soutenu en publiciste et en guerrier la cause serbe. Jacques Mathyer, ancien directeur et professeur honoraire de l'Institut de police scientifique et de criminologie de Lausanne, lui consacre une monographie anecdotique qui suit les sources plutôt que les critiquer pour construire son objet (2000, Institut de police scientifique et de criminologie, Faculté de droit, Université de Lausanne, série criminalistique XVII), 208 p., nombreuses illustrations. Précédé d'une brève introduction, divisé en neuf courts chapitres (les six premiers concernent Reiss criminologue), bouclé par une "Conclusion » sous forme d'un portrait intime, enrichi d'annexes (chronologie de l'Institut de police scientifique dès la démission en 1919 de Reiss à aujourd'hui; bibliographie de Reiss), sans index onomastique ni bibliographie, l'ouvrage de Mathyer n'est pas la première étude sur Reiss, familier du criminologue lyonnais Edmond Locard ${ }^{4}$. Son nom est attaché à celui de l'Université de Lausanne, la première au monde à donner jusqu'à aujourd'hui une formation académique de police scientifique ( Sciences forensiques»). Comme Hippolyte Gosse à Genève, Hans Gross à Vienne ou Alexandre Lacassagne à Lyon, Reiss incarne les mutations socio-culturelles dans l'institutionnalisation de la criminologie à la fin du XIXe siècle : chimiste passant de la photographie scientifique à la photographie judiciaire, puis à la police scientifique utilisée par l'État. Né dans le grand-duché de Bade au sein d'une famille de notables juifs convertis au protestantisme, Reiss mène des études scientifiques, émigre en 1893 à Lausanne, y obtient en 1898 un doctorat ès sciences. Photographe à ses heures, il participe au lancement la même année du Journal suisse de photographie. Chef des travaux photographiques de l'Université de Lausanne 
(1899), donnant des conférences sur la « photographie scientifique ", il est attiré par le bertillonage introduit à Berne, Genève et Lausanne. Vers 1900, il travaille à Paris sous la direction de Bertillon. Privat-docent dès 1902 en "photochimie " à la Faculté des sciences de l'Université de Lausanne, il enseigne la "photographie judiciaire et la photographie signalétique ", et publie son fameux La Photographie judiciaire (Paris, 1903). Marqué par l'anthropométrie judiciaire qui gagne l'Europe entière, obtenant (1901, 1903 ?) le diplôme de "Signalement descriptif » décerné par la Préfecture de police de Paris, il édite en 1905 son Manuel du portrait parlé à l'usage de la police. Après avoir été chargé des services de radiographie et de radioscopie de l'Hôpital cantonal depuis 1904, Reiss est nommé en 1906 professeur extraordinaire de «sciences photographiques et policières "; sa leçon inaugurale (in Archives d'anthropologie criminelle de Lacassagne) consacrée aux «méthodes scientifiques dans les enquêtes judiciaires et policières » actualise son projet normatif qui le placera à la tête de l'Institut de Police scientifique de l'Université de Lausanne créé en 1909, à son initiative, sous la houlette scientifique des Facultés de droit, de médecine et de sciences. Menant et équipant son institut, dirigeant ses collaborateurs, formant des étudiants et des magistrats suisses ou étrangers, Reiss devient un expert connu, recherché sur la plan national et international. Chevalier de la légion d'honneur en 1911, prononçant des conférences en Russie (1912), au Brésil (1913), il participa à la création d'un service dactyloscopie à la Sûreté vaudoise (1913). Dès septembre 1914, il gagne plusieurs fois la Serbie pour étudier médico-légalement les «atrocités austrohongroises » qu'il relate dans la presse suisse. Sur le front des Balkans durant la Grande Guerre, Reiss démissionne de sa charge en octobre 1919 au profit de son élève Marc Bischoff, autre figure emblématique de la police scientifique. Installé à Belgrade jusqu'à sa mort en 1929, vivant "modestement" de son travail d'expert, Reiss sécurise notamment la nouvelle monnaie nationale.

6 L'histoire sociale et culturelle de la criminologie enrichit celle du crime et de la justice. Si cette monographie documente le contexte lausannois de l'institutionnalisation des travaux d'un "pionnier de la police scientifique", elle reste peu analytique sur les enjeux scientifiques, sociaux, juridiques et politiques de la criminologie héritière de la médecine légale du $\mathrm{XIX}^{\mathrm{e}}$ siècle. Elle néglige l'horizon d'attentes positiviste et sécuritaire de la "science du crime", située au carrefour des sciences expérimentales et des pratiques sociales normatives. Au temps de Reiss, dans le contexte de la "défense sociale " d'Adolphe Prins et liée à la Sûreté, l'internationale des criminologues n'obéit pas qu'à des considérations techniques de laboratoire. Attachés au contrôle social des suspects et à la répression des délits dans un État de droit, les objectifs de la criminologie positiviste sont précis : armer scientifiquement la police, signaler les délinquants, neutraliser les récidivistes, quantifier les traces et les indices sur la scène du crime, surveiller les réfugiés et les « révolutionnaires ». Pourquoi ne pas dire que la majorité des experts que Reiss forme à Lausanne proviennent d'États autoritaires (Russie tsariste; Roumanie, etc.) qui traquent leurs opposants? Mathyer est hagiographe : il décrit la "biographie exemplaire» de Reiss sans démontrer que sa rapide ascension sociale signale le triomphe politique de la criminologie avant 1914. On ignore tout des idées de Reiss sur la causalité du crime alors fortement débattue : est-il lombrosien (criminel né) ? Suit-il Lacassagne (milieu) ? Offre-t-il une synthèse de ces deux doctrines? Mathyer ne nous dit pas pourquoi, ni comment le criminologue positiviste révolutionne certainement la police scientifique de son temps. Une police scientifique bientôt dominante, autant sur la scène du crime social, que sur celles de 
l'activisme politique, des guerres et des accidents individuels ou de masse. Le chapitre consacré au "moment serbe " de Reiss ne repose que sur des sources favorables au policier scientifique, récompensé par des obsèques nationales à Belgrade. Finalement, Mathyer peut-il véritablement écrire l'histoire d'une institution de police scientifique dont il a été le directeur ? Il reviendrait plutôt à des chercheurs indépendants, attachés à l'éthique de l'historien, de mener de telles enquêtes en accédant aux archives de la police. Ceci est un enjeu capital dans le champ toujours plus large de l'histoire du crime et des institutions pénale pour le $\mathrm{XX}^{\mathrm{e}}$ siècle. Policier de formation oubliant que des sources dépourvues de questionnaire initial ne font pas l'histoire, l'auteur s'enferme dans leur logique institutionnelle et positiviste. Ignorant l'historiographie actuelle sur le sujet, il célèbre, comme l'historiographe du roi de l'Ancien régime, l'histoire édifiante de son héros à travers celle des «progrès » de la criminalistique née avec le $\mathrm{XX}^{\mathrm{e}}$ siècle.

7 Si dès le XIX ${ }^{e}$ siècle, le « criminologue » incarne ainsi la science positiviste du crime, le "criminaliste» (néologisme en français dès 1660) est une figure classique de la modernité judiciaire née au XVI ${ }^{\mathrm{e}}$ siècle lorsque l'État absolu monopolise le droit de punir en renforçant sa souveraineté autour des droits régaliens. Benedict Carpzov (1595-1666), Giulio Claro (1525-1575), Prospero Farinacci (1544-1618), Jean Imbert, Daniel Jousse (1704-1781): dans toute l'Europe, ces juristes bien connus de l'Âge classique systématisent notamment le droit pénal selon les sources de la loi (statuts, coutumes, Édits, ordonnances, etc.). Ils élaborent une doctrine pénale qui unifie dans la pratique l'Europe continentale autour d'un droit criminel relativement commun. Partout, l'homo criminalis est alors considéré comme un pécheur à neutraliser par une peine infamante et expiatoire ${ }^{5}$. C'est à Jean Domat (1625-1695), un "criminaliste oublié » qui publie en 1689 à Paris une somme juridique, maintes fois rééditée au siècle des Lumières, Les Lois civiles dans leur ordre naturel, suivies du Droit public, qu'est consacrée la monographie érudite de Claudio Sarzotti (2001). Entre histoire des idées, philosophie du droit et histoire de la culture juridique, cette élégante monographie est divisée en deux parties. La première section (pp. 1-70) est divisée en quatre objets : «Domat, criminaliste oublié »; place du droit pénal dans le droit public; typologie pénale selon Domat; la peine comme instrument de politique criminelle ante litteram; l'objectif répressif des peines dans le mécanisme de la prévention générale du crime. La seconde partie contient une longue note méthodologique (pp. 73-90) ainsi que la traduction italienne du fameux chapitre «Des crimes et des délits» (pp. 91-162) qui constitue le livre III de la section consacrée par Domat au Droit public. Jean Domat n'est pas un pénaliste. Romaniste et civiliste, il élabore une « science juridique » pour penser le droit privé comme matrice du droit public de l'État moderne. En 1689, la publication de son énorme traité (près de 900 pages dans l'édition in-folio éditée en 1702 à Luxembourg) le place, avant Jean Pothier (1699-1772) qui systématisa le droit civil de la monarchie moderne, comme le " précurseur » du Code civil napoléonien de 1810. Ancré dans une culture juridique chrétienne, Domat privilégie le droit civil comme objet de réflexion juridique, notamment parce qu'il valorise les «lois naturelles» ou « immuables » auxquelles les individus obéissent grâce à leur « sociabilité » naturelle : amour pour Dieu et pour leur prochain. Le noyau fondamental de la société étant la famille et ses normes civiles, le reste de l'appareil normatif qui en découle n'a pas besoin d'être trop encadré par des lois positives ou "arbitraires». Comme est "naturel » l'effort des hommes pour éviter le péché, l'obéissance au roi, source de la loi, est «naturelle». Elle repose sur une triple norme: droit divin (le roi est le 
lieutenant sacré de Dieu sur terre); droit naturel (le roi est le père de tous ses sujets); droit politique (selon les lois fondamentales du royaume, il incarne la souveraineté de l'État). Puisque le crime contre le roi ou contre les particuliers résulte du péché et de la violation des "lois naturelles", aucune législation positive ne peut le prévenir. En douze pages de son ouvrage in-folio, Domat fait donc le tour de la problématique des "Crimes et des délits » qui méritent peu de règles. Il aborde le droit de punir en suivant la conception classique de la qualification du crime selon ses «différentes circonstances " morales et matérielles. S'y ajoute la motivation de la peine proportionnelle au péché du criminel selon les impératifs de la prévention générale nécessaire à maintenir l'ordre public dans une société de pécheurs où chacun glisse sur la « pente universelle qui porte les hommes au mal » prémédité ou non (« contenir par la vue et la crainte des peines »). Basée sur la poursuite d'office (Parquet), tournée vers les peines classiques (mort, galères, bannissement), la justice pénale selon Domat est une " police conforme à l'esprit de la Religion Chrétienne ». Sécularisée dans sa forme inquisitoire mais suivant la morale du christianisme dans son "esprit », elle poursuit les crimes de « lèse-majesté divine », les « attentats contre le Prince et contre l'État » et les délits matériels ou de sang contre les individus (vols, meurtres, etc.). En découle une quadruple pénalité harmonisée selon la gravité du crime-péché né de l'« oisiveté » ou de la "pauvreté » : pécuniaire, infamante, corporelle et capitale. Surtout éliminatrice, la pénalité sera préventive contre la récidive : corriger et «avertir » les criminels de «changer de vie». Pourtant, malgré les supplices publics, le système pénal est impuissant face à l'homme pécheur dévoré de "passions» et d'«inclinations " criminogènes. Attaché à la déterrition du crime par le mal de la peine, Domat suit pourtant le scepticisme du «criminaliste» parisien Jean De Mille (fin $\mathrm{XV}^{\mathrm{e}}$ s.-1563) ${ }^{6}$ : " ainsi ceux que l'avarice a engagé dans l'habitude du larcin, dérobent aux spectateurs [...] pendant le supplice » d'un voleur. Minimaliste dans sa formulation, le système pénal de Domat est une miniature de celui de l'âge classique, profondément providentialiste. Il valide l'idée que l'ordre social protégé par la justice assure hic et nunc l'ordre idéal du bien voulu par Dieu, qui a « pourvu par une autre conduite de sa Loi divine dans la police à corriger ou réprimer ceux que l'esprit de la Religion ne corrige point». La justice n'est donc aux yeux de Domat que la terrestre "police conservant l'esprit universel de la Loi divine pour le bien commun de la société ». La philosophie pénale de Domat est un ingrédient de l'art de gouverner tel que le concevaient les doctrinaires-pénalistes de la fin du XVII ${ }^{\mathrm{e}}$ siècle et du siècle des Lumières. Pour Sarzotti, la question de la sécularisation du droit de punir est licite chez Domat dans son jusnaturalisme logico-déductif et cartésien. Or, n'oublions pas qu'il soude le droit naturel et le providentialisme, à l'instar de son contemporain Bossuet qui énonce une doctrine chrétienne de l'absolutisme selon les saintes écritures. Face à l'œuvre du civiliste, Montesquieu, oublié ici par Sarzotti, ne s'y trompera pas. En réfutant le providentialisme de Domat, il prendra pourtant l'idée de son titre (De l'Esprit des lois) dans le chapitre IV des Lois civiles dans leur ordre naturel consacré aux «engagements » civils définis par un droit positif qui sécularise les objectifs de la providence divine («esprit des lois», une dizaine d'occurrence). Pour Montesquieu, l'esprit des lois réside dans l'histoire des sociétés, dans l'évolution des mœurs, dans le volontarisme législatif des États : au pénal comme au civil, le juge est alors la bouche de la loi des hommes. Pour Domat, l'esprit des lois réside dans celui de la religion : le juge ne "doit rendre compte qu'à Dieu seul». Le providentialisme domatien perdra son actualité au siècle des Lumières avec la sécularisation progressive du droit de punir et 
son enracinement dans la causalité sociale du crime. Pourtant, viscéralement attaché à un droit pénal servant la monarchie de droit divin, le grand «criminaliste » PierreFrançois Muyart de Vouglans (1723-1791), hostile à la conception rousseauiste du contrat social, adversaire acharné de l'abolitionnisme beccarien et de la modération pénale d'un Montesquieu, réhabilite le providentialisme juridique de Jean Domat avec la publication en 1780 de ses Lois criminelles de France dans leur ordre naturel. L'intéressante monographie de Sarzotti souligne ainsi les continuités et les ruptures épistémologiques dans la conception d'un droit pénal moderne à l'orée du siècle des Lumières. Mis dans la main divine prolongée par celle du souverain absolu, l'État justicier de Domat s'effacera progressivement devant l'État de droit défini par des lois positives illustrant la volonté générale du souverain collectif qu'est le peuple.

8 L'histoire culturelle et institutionnelle du droit pénal moderne est liée aux formes prises dès la fin du Moyen Âge par l'enquête judiciaire qu'induit la procédure inquisitoire dans toute l'Europe continentale de l'Ancien régime. Enseignant l'histoire du droit pénal à l'Université Frédéric II de Naples, Giorgia Alessi consacre son dernier essai érudit, à la naissance et aux avatars de l'enquête judiciaire entre la fin du Moyen Âge, via les Lumières et la Révolution française, et la formation des États-nations européens, jusqu'à l'adversary system étasunien au XX $\mathrm{X}^{\mathrm{e}}$ siècle (2001, index onomastique, pp. 269-276). Précédés d'une «introduction» méthodologique exposant, malgré l'inflation des études, les enjeux contemporains de continuer à « raconter » le judiciaire pour comprendre l'histoire de l'État moderne et contemporain, cinq chapitres denses éclairent dans la longue durée les racines européennes des formes actuelles de la poursuite pénale (délits et compositions, enquête ou les juges itinérants, appel ou les grandes ordonnances de l'époque moderne, procès et opinion publique au XVIII ${ }^{e}$ siècle, instruction secrète, interrogatoire). L'enquête judiciaire signale la modernité de la légalité du droit criminel qui aujourd'hui fonde la justice. Vaste champ, vaste temporalité, figures multiples de pénalistes ou criminologues évoqués (Beccaria, Bertillon, Muyart de Vouglans, etc.), discussion historiographique soutenue (Carbasse, Foucault, Sbriccoli), objet complexe en raison de l'ampleur de l'enquête : l'ouvrage est dense. Il montre que l'histoire de l'État moderne - de l'État justicier à l'État de droit ne peut plus se penser hors de celle du droit de punir, ainsi que de tous les savoirs judiciaires et criminologiques qui en sont issus.

9 Même thèse politique dans l'ouvrage collectif publié sous la direction de Xavier Rousseaux et René Lévy (1997), biographies des vingt-six auteurs (historiens, sociologues), sans index. L'histoire de l'État moderne recoupe celle du droit de punir. Aujourd'hui, la difficulté de fonder un droit pénal européen provient certainement du fait que le droit de punir est au cœur de la souveraineté étatique. Dès la fin du Moyen Âge, les sociétés occidentales se sont progressivement modernisées et développées grâce au processus de "pacification " généré par le monopole pénal accaparé et exercé par l'État selon des modalités, des chronologies et des mises en formes institutionnelles diverses. Pour le sociologue allemand Norbert Elias, ce processus était celui de la " civilisation » qui, autour du modèle curial, contraignait l'individu moderne à raboter ses pulsions, à renoncer à la brutalité interpersonnelle, à apprendre des normes éducatives (pensons à la Civilité puérile d'Erasme de Rotterdam), à intérioriser des codes de maintien corporel et alimentaire qui modifiaient sa sensibilité en le raffinant et élevant ses seuils d'intolérance face à la violence criminalisée par l'État. Cette reconfiguration des sensibilités mise à l'ordre du jour par la culture néo-platonicienne et humaniste de la Renaissance constitue le cadre humain des mutations pénales qui 
vont labourer l'Europe continentale avec l'avènement des monarchies absolutistes de droit divin qui placent le monopole de la poursuite au cœur de leur souveraineté. Comme l'avait notamment montré Jean Bodin en 1576 dans La République, l'exercice de la souveraineté implique l'obligation de la poursuite pénale; celle-ci contraint à prohiber la justice privée, à affirmer la puissance du ministère public («procureur fiscal », " procureur du roi ", " procureur général », etc.), à édifier un corpus législatif unifié et rédigé en langue vernaculaire pour contrer les coutumes, à établir une pénalité non compensatoire et expiatoire (du talion au gibet) ou encore, sur le plan procédural, à institutionnaliser la procédure inquisitoire (orale, secrète, basée sur l'aveu et l'expertise comme système de preuves) qui rationalise les processus d'incrimination de l'homo criminalis. Tel est l'essentiel de la lente construction d'une nouvelle culture juridique et judiciaire qui ordonne les pratiques pénales en Europe moderne sous le régime arbitraire des délits et des peines, autant dans les régimes absolutistes que dans les États républicains. Préparé par les « codifications pénales de l'Ancien régime » qui consolident le monopole de la poursuite étatique, le passage vers la légalité, qu'universalise en Europe continentale le Code pénal français de 1791 et de 1810, constitue le point d'aboutissement de l'alliance indéfectible entre l'État et le droit de punir.

10 C'est à l'histoire de cette alliance, qui en Europe, a fondé notre modernité répressive et la sécurité des individus, qu'est consacré cet ouvrage, ouvert par une introduction des éditeurs scientifiques qui invitent à lire vingt-trois contributions rédigées en français et en anglais par autant d'historiens européens de la poursuite pénale. Les contributions résultent de travaux présentés entre 1992 et 1994 au séminaire européen ( Les États et le pénal : acculturation juridique et intégration nationale ») qui s'est tenu aux Facultés universitaires de Saint-Louis de Bruxelles. Allemagne, Angleterre, Italie, France, Pays-Bas, Corse, Belgique, Flandres et Wallonie : si la géographie européenne de l'ouvrage est aussi étendue que sa chronologie allant du Moyen Âge à l'époque contemporaine (XIII $-\mathrm{XX}^{\mathrm{e}}$ siècles), l'objet étudié (soit le rapport croissant entre l'État et le pénal) donne la cohérence à l'ensemble dans deux sections (« Acculturation juridique et intégration nationale »; "Les vecteurs de l'acculturation: droit et institutions »). Justice du roi en France à la fin du Moyen Âge, exception du modèle hollandais dans la genèse d'une justice moderne et étatique, codifications au siècle des Lumières et au $\mathrm{XIX}^{\mathrm{e}}$ siècle, maréchaussée, territoire judiciaire, ministère public : parmi d'autres, ces objets minutieusement étudiés dans l'ouvrage en forgent la bonne unité thématique. Ils renforcent la thèse de ses éditeurs scientifiques: il n'est pas possible de penser l'histoire de l'État moderne en la détachant du droit de punir qui fonde sa souveraineté en pacifiant les sociétés européennes et en menant, via l'État de droit, à la démocratie. L'histoire du droit de punir en Europe moderne et contemporaine ne peut donc qu'être comparatiste. Comme le montre bien cet ouvrage, elle implique une collaboration plus étroite entre les disciplines de l'histoire, de la sociologie et celles du droit, souvent trop polarisées par l'Université. 


\section{BIBLIOGRAPHIE}

Alessi, G., Il processo penale. Profilo storico, Roma-Bari, Laterza, 2001.

Artières, Ph., Clinique de l'écriture. Une histoire du regard médical sur l'écriture, Luisant, Les empêcheurs de penser en rond, 1998.

Artières, Ph., Livre des vies coupables, Paris, Albin Michel, 2002.

Crépieux-Jamin, J., Les éléments de l'écriture des canailles, Paris, 1923.

Laingui, A., L'homme criminel dans l'ancien droit, Revue de science criminelle et de droit pénal comparé, 1983, janvier-mars, 1, pp. 15-35.

Levental, Z., Rodolphe Archibald Reiss, criminaliste et moraliste de la Grande Guerre, Lausanne, L'Âge d'Homme, 1992 (traduit du serbo-croate).

Mathyer, J., Rodolphe A. Reiss, Pionnier de la criminalistique. Les années lausannoises et la fondation de l'Institut de police scientifique et de criminologie, Lausanne, Payot, 2000.

Mille, J. de, Pratique criminelle [1541], Moulins, 1983.

Porret, M., Médecine du crime, médecine de l'âme, Crime, Histoire et Sociétés, 1998, 2, 2, pp.

121-126.

Rousseaux, X., Lévy, R., Le pénal dans tous ses États. Justice, États et sociétés en Europe (XII ${ }^{e}-X X^{e}$ siècles), Bruxelles, publications des Facultés universitaires Saint-Louis, 1997.

Sarzotti, C., Domat criminalista, Milan, Padoue, CEDAM, 2001.

\section{NOTES}

1. Revue publiée par l'Association pour l'histoire de l'éducation surveillée et de la protection judiciaire des mineurs, ainsi que le Centre national de formation et d'études de la protection judiciaire de la jeunesse; numéro hors-série publié à l'adresse du ministère de la Justice avec le soutien du GIP Mission de recherche droit et justice.

2. Porret (1998), «Forum » bibliographique.

3. Cf. Crépieux-Jamin (1923).

4. Levental (1992).

5. Cf. le bel article déjà ancien d'A. Laingui (1983).

6. Mille (1983).

\section{AUTEUR}

\section{MICHEL PORRET}

Université de Genève, michel.porret@lettres.unige.ch 